\title{
Thoracic epidural catheter for postoperative pain control following an ineffective transversus abdominis plane block using liposome bupivacaine
}

This article was published in the following Dove Press journal: Journal of Pain Research

16 January 2017

Number of times this article has been viewed

\author{
Brian D Terrien' \\ David Espinoza ${ }^{2}$ \\ Charles C Stehman ${ }^{3}$ \\ Gabriel A Rodriguez' \\ Nicholas C Connolly' \\ 'Department of Anesthesiology, Naval \\ Medical Center San Diego, ${ }^{2}$ Surface \\ Warfare Medical Institute, San Diego, \\ ${ }^{3}$ Department of Anesthesiology, \\ Robert E. Bush Naval Hospital, \\ Twenty Nine Palms, CA, USA
}

\begin{abstract}
A 24-year-old female with a history of ulcerative colitis underwent colectomy. The patient received an ineffective transversus abdominis plane (TAP) block with liposome bupivacaine (Exparel) intraoperatively and was started on a hydromorphone patient-controlled analgesia 5 hours after the TAP block, which did not relieve her pain. A continuous thoracic epidural (CTE) was then placed after blood levels of bupivacaine were drawn, and the patient immediately experienced significant pain relief. The combined use of liposome bupivacaine and bupivacaine CTE infusion in the postoperative management of this patient demonstrated no safety concerns, provided excellent analgesia and plasma concentrations of bupivacaine remained far below toxic levels.
\end{abstract}

Keywords: liposome bupivacaine (bupivacaine liposome injectable suspension), plasma bupivacaine levels, transversus abdominis plane (TAP) nerve block, thoracic epidural

\section{Background}

Annually, in the US, there are approximately 45 million surgeries performed on inpatients $^{1}$ and an additional 55 million surgical and nonsurgical procedures performed in the ambulatory setting. ${ }^{2}$

Inadequate postsurgical pain control can lead to an increased incidence of surgeryrelated complications such as infection, poor wound healing, or increased risk of deep vein thrombosis due to delays in ambulation and mobility. This all leads to an increase in costs and resources due to longer hospital stays and more frequent readmissions., A balanced approach to postsurgical pain management often seeks to improve analgesia effectiveness while minimizing opioid use and the possibility for opioid-related adverse events. ${ }^{4}$

As part of a multimodal pain control plan, local anesthetics continue to be used frequently and safely in the perioperative period. Their duration of action is relatively short, and adjuncts such as epinephrine, clonidine, or dexamethasone are often used to extend the analgesic benefits, normally with the goal of minimizing opioid consumption. Liposome bupivacaine is a relatively new treatment option for the management of postoperative pain that can potentially extend the duration of action of a single injection to 96 hours. $^{5}$

With liposome bupivacaine, the bupivacaine is encapsulated by a lipid membrane made up of polyhedral particles composed of numerous internal aqueous chambers known as DepoFoam (Figure 1). ${ }^{5}$ The bupivacaine is unaltered and is released over a period of time due to erosion and/or reorganization of the lipid membranes. ${ }^{6}$
Correspondence: Brian D Terrien Department of Anesthesiology, Naval Medical Center San Diego, 34800 Bob Wilson Drive, San Diego, CA 92134 , USA

Tel + I 6193037258799

Email brian.terrien@gmail.com 


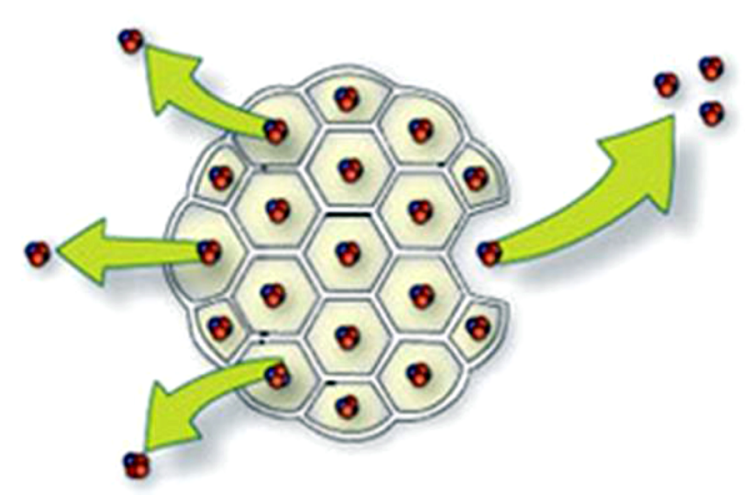

Figure I Cartoon depiction of DepoFoam surrounding bupivacaine. ${ }^{6}$

Liposome bupivacaine was approved by the US Food and Drug Administration (FDA) in October 2011 for single-dose administration into surgical sites. Liposome bupivacaine has shown superiority in postoperative pain management following various surgical procedures (eg, inguinal hernia repair) by not only reducing cumulative pain scores at 72 hours but also by reducing the use of opioids, as well as secondary complications. ${ }^{7,89}$ The FDA package insert for liposome bupivacaine specifically warns against the use of additional bupivacaine and other amide-type local anesthetics for 96 hours after the use of liposome bupivacaine because of potentially additive toxic effects ${ }^{5}$ such as local anesthetic toxicity. While the use of liposome bupivacaine in both peripheral nerve blocks and in the surgical wound is a growing area of interest, there is little research to date on the combined use of liposome bupivacaine and local anesthetics for pain control and its safety or efficacy.

Transversus abdominis plane (TAP) blocks are one option for the administration of local anesthetics in postoperative pain management. Evidence-based data regarding the pharmacokinetic profile of liposome bupivacaine infiltrated into the TAP are lacking. Although there are no studies to date looking specifically at TAP blocks, previous studies evaluating liposome bupivacaine for other types of peripheral never blocks have measured plasma concentration at 12-24 hours or at approximately 36 hours postoperatively. ${ }^{10,11}$ In this report, we describe the safety and effectiveness of a thoracic epidural placed 12 hours following the TAP administration of liposome bupivacaine in a patient after an open colectomy.

\section{Case report}

A 24-year-old female with a history of ulcerative colitis, gastritis, hemorrhoids, and gastroesophageal reflux disease was admitted for an ulcerative colitis flare and subsequently underwent colectomy. The patient's surgical history consisted of tonsillectomy and adenoidectomy 12 years earlier, as well as multiple esophagogastroduodenoscopies and colonoscopies over the past 4 years. Patient medications prior to surgery included mesalamine, and infliximab for management of her ulcerative colitis.

The patient underwent laparoscopic colectomy, which was converted to an open colectomy. During the intraoperative period, the patient received acetaminophen 1,000 $\mathrm{mg} I V$, fentanyl $350 \mu \mathrm{g}$ IV, hydromorphone $2 \mathrm{mg} \mathrm{IV}$, and lidocaine $50 \mathrm{mg}$ IV ( $>7$ hours prior to administration of liposome bupivacaine) as part of a multimodal pain management plan. Upon completion of the procedure and closure of the midline abdominal incision, the anesthesia team placed bilateral subcostal TAP blocks with a total of $266 \mathrm{mg}$ of liposome bupivacaine using ultrasound guidance. Overnight, the patient endorsed 10/10 pain, and $\sim 5$ hours after liposome bupivacaine infiltration, a hydromorphone patient-controlled analgesia (PCA) pump was started. The patient received an initial bolus of $0.8 \mathrm{mg}$ hydromorphone in addition to acetaminophen $1,000 \mathrm{mg} \mathrm{IV}$. The patient endorsed $9 / 10$ pain throughout the remainder of the night, receiving only minimal pain relief with the PCA.

On postoperative day (POD) 1, the surgeon requested that the regional anesthesia team consider placing a thoracic epidural because the patient continued to have inadequate pain control and was unable to ambulate or sit on a chair. Prior to epidural placement, a total plasma bupivacaine concentration was drawn and sent to an outside testing facility. The result was not immediately available; however, 3 days later it was reported at $<0.2 \mu \mathrm{g} / \mathrm{mL}$, falling at the low end of the laboratory reference range of $0.1-4.0 \mu \mathrm{g} / \mathrm{mL}$. A continuous thoracic epidural (CTE) was placed at the level of T7-T8, 12.5 hours after the TAP block was administered. The CTE was tested and then infused with $8 \mathrm{~mL}$ of $2 \%$ lidocaine bolus, resulting in a significant reduction in her pain score.

One hour after successful CTE placement, an epidural infusion of bupivacaine $0.1 \%$ with hydromorphone $10 \mu \mathrm{g} / \mathrm{mL}$ was started at $6 \mathrm{~mL} / \mathrm{h}$. Patient-controlled epidural anesthesia bolus was not permitted due to concern for potential local anesthetic systemic toxicity if escalating doses of local anesthetics were used. Three hours later, the epidural infusion was no longer providing adequate analgesia. Following discussion of safety concerns with the pharmacist, an additional $5 \mathrm{~mL}$ bolus of bupivacaine $0.1 \%$ with hydromorphone $10 \mu \mathrm{g} / \mathrm{mL}$ was administered with good relief (the patient reported a reduction of pain from $8 / 10$ to $3 / 10$ ).

On POD 2, 34 hours after the TAP block was placed and 20.5 hours after the start of the bupivacaine/hydromorphone epidural infusion, the patient's plasma bupivacaine concentration was $0.7 \mu \mathrm{g} / \mathrm{mL}$. The patient's pain remained 
controlled overnight. The next morning, she was able to get out of bed and sit on a chair and expressed a desire to begin walking. The patient has provided informed consent for the publication of this case report.

\section{Discussion}

Liposome bupivacaine's prescribing information warns that the use of liposome bupivacaine followed by other bupivacaine formulations has not been studied in clinical trials and these other bupivacaine formulations should be withheld for 96 hours. ${ }^{5}$ The concern is the significant systemic plasma concentrations of bupivacaine, which can persist for 96 hours (Figure 2).

Elevated levels of bupivacaine can lead to bupivacaine toxicity and if levels are high enough, there is a greater incidence of cardiotoxicity compared to other local anesthetics. ${ }^{12}$ Lightheadedness, dizziness, tinnitus, vision problems, and drowsiness may be the initial central nervous system manifestations. Physical signs and symptoms often present as shivering, tremors, or twitching and can even lead to generalized tonic-clonic convulsions. If levels are high enough in the central nervous system, hypoventilation and apnea may occur. Since it is lipid soluble with a very high degree of protein binding, it has a long duration of action. It also avidly binds to cardiac sodium channels and does not release from its binding site quickly like other local anesthetics, which then can create dysrhythmias at lower concentrations than less potent local anesthetics. Treatment for potential cardio- vascular collapse focuses on the administration of intralipid solution to first remove the bupivacaine from the binding sites so that advanced cardiac life support can then be effective. ${ }^{12}$

Some studies ${ }^{7,13}$ have examined the systemic plasma levels of liposome bupivacaine during isolated use, but no studies were found where local anesthetic levels were measured when using both liposome bupivacaine and amide local anesthetic. In a study looking at postsurgical pain in total knee arthroplasty, liposome bupivacaine was compared to bupivacaine hydrochloride $(\mathrm{HCl})$. The maximum observed plasma concentration of liposome bupivacaine $266 \mathrm{mg}$, measured at 24 hours, was $0.340 \mu \mathrm{g} / \mathrm{mL} .{ }^{13}$ Jorfeldt et al concluded that plasma concentrations of bupivacaine of $4 \mu \mathrm{g} / \mathrm{mL}$ or greater have been associated with systemic toxicity. ${ }^{14}$ In another study using Rhesus monkeys, after a continuous IV infusion, the seizure threshold was determined to be a plasma bupivacaine concentration of $4.5 \mu \mathrm{g} / \mathrm{mL}( \pm 1.7) .{ }^{15}$

The maximum plasma concentration of bupivacaine measured after a 21-hour epidural infusion study on 21 healthy volunteers of a $0.25 \%$ solution was $0.90 \mu \mathrm{g} / \mathrm{mL}$ (Figure 3 ). ${ }^{16}$ Again, in our case report, $0.1 \%$ bupivacaine was used for the epidural infusion, much lower than the concentration studied by Emanuelsson et al, but ours was also continued until POD 4.

Based on the studies described earlier, the sum of the maximum plasma concentrations of liposome bupivacaine $266 \mathrm{mg}$ and an epidural infusion of bupivacaine should be far less than the threshold for systemic toxicity.

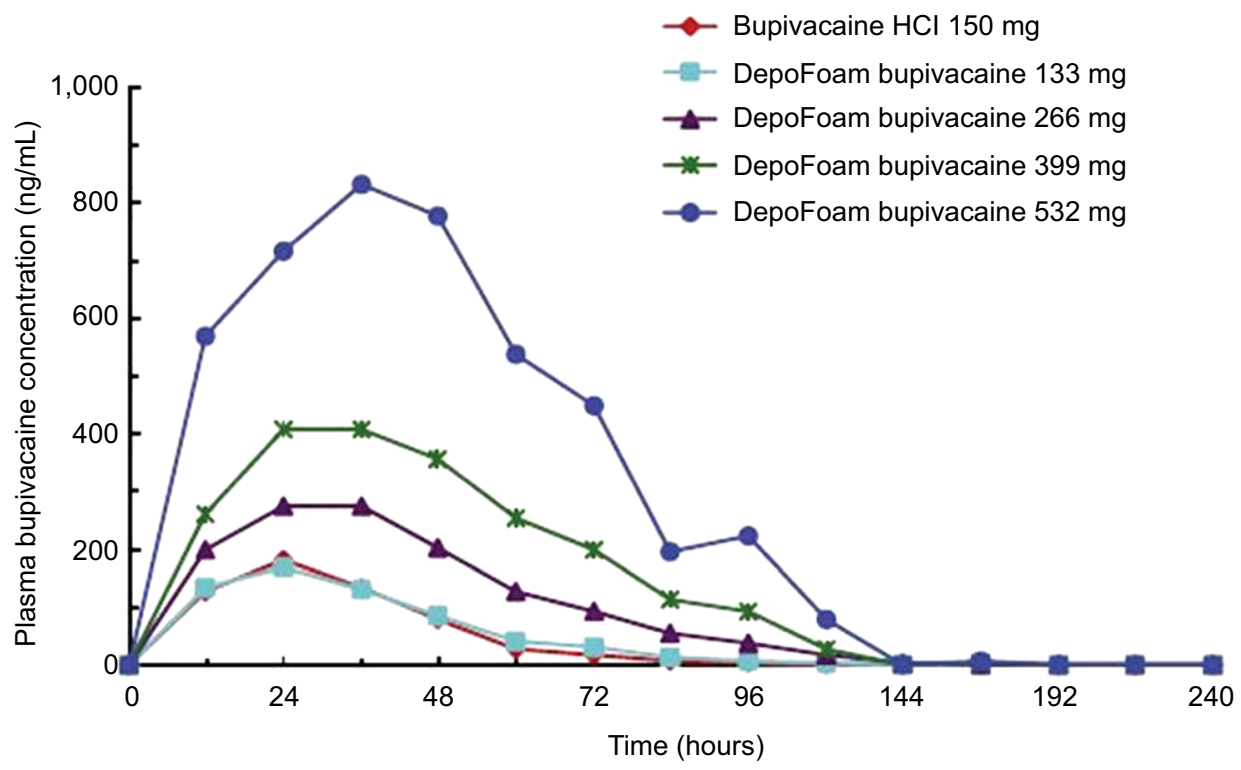

Figure 2 Mean plasma bupivacaine concentrations after administration of DepoFoam bupivacaine or bupivacaine hydrochloride by infiltration to patients undergoing total knee arthroplasty.

Note: Reprinted from Knee, 19(5), Bramlett K, Onel E, Viscusi ER, Jones K, A randomized, doubleblind, dose-ranging study comparing wound infiltration of DepoFoam bupivacaine, an extended-release liposomal bupivacaine, to bupivicane $\mathrm{HCl}$ for postsurgical analgesia in total knee arthroplasty, 530-536, Copyright 20I2, with permission from Elsevier. doi: 10.1016/j.knee.2011.12.004.13 

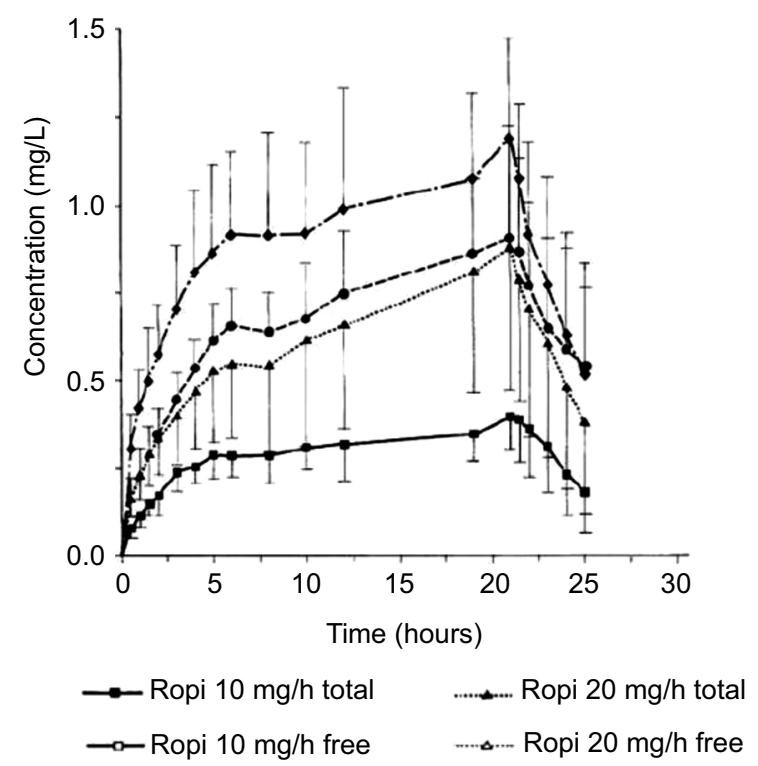
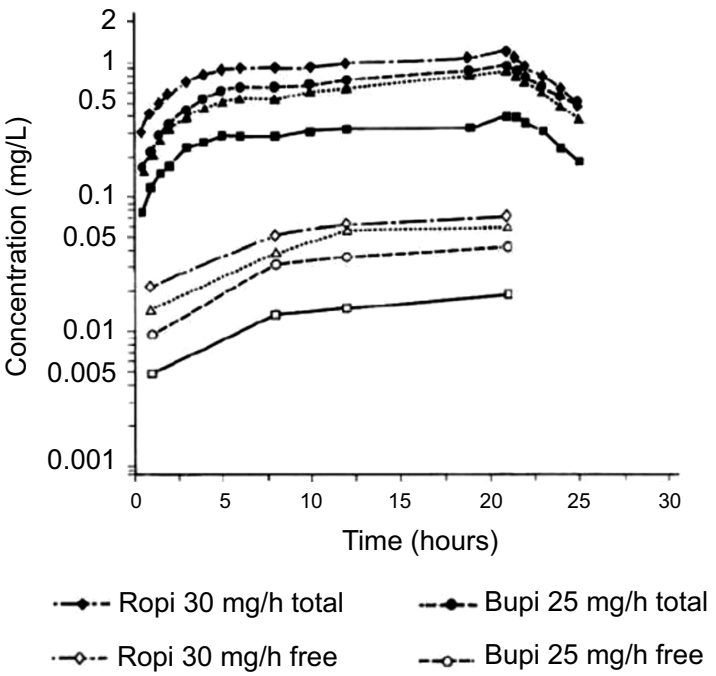

Figure 3 Mean total and free plasma concentrations after continuous epidural infusion of 10,20 , and $30 \mathrm{mg} / \mathrm{h}$ ropivacaine and $25 \mathrm{mg} / \mathrm{h}$ bupivacaine over $2 \mathrm{I}$ hours. Note: Reproduced from Emanuelsson BM, Zaric D, Nydahl PA, Axelsson KH. Pharmacokinetics of ropivicaine and bupivacaine during 21 hours of continuous epidural infusion in healthy male volunteers. Anesth Analg. 1995;8I(6): II63-1168. ${ }^{16}$ Promotional and commercial use of the material in print, digital or mobile devise format is prohibited without the permission from the publisher Wolters Kluwer. Please contact healthpermissions@wolterskluwer.com for further information.

Abbreviations: Ropi, ropivacaine; Bupi, bupivacaine.

A similar case of liposome bupivacaine use in conjunction with an amide local anesthetic was conducted in our institution a year prior to this case report where we documented safe, simultaneous use of liposome bupivacaine in the surgical wound along with an epidural infusion of bupivacaine. ${ }^{17}$ It was hoped that using both an epidural and infiltrating the surgical wound would provide a multimodal, balanced, and adequate means of minimizing the use of opioids and their potential adverse events. A thorough literature review revealed no cases where liposome bupivacaine and an epidural were used simultaneously. In that case, postoperative pain scores never exceeded 2/10. There were never concerns for local anesthetic systemic toxicity, there was minimal use of postoperative IV narcotics, and there were no other adverse events or complications with the pain regimen.

The patient was discharged from the intensive care unit POD 2, was eating a regular diet, and started physical therapy all within days of her procedure. Her pain remained well controlled while on the ward and the epidural was discontinued on POD 4. Although plasma concentrations of bupivacaine were not drawn, it was postulated that they would remain far below the threshold for toxicity, based on the studies described earlier. ${ }^{13,16}$

In this case report, the patient received a TAP block with liposome bupivacaine that did not provide adequate pain relief and was then started on a hydromorphone PCA, which also barely alleviated her pain. A large portion of the pain that patients experience after abdominal surgery is derived from the abdominal wall incision. A TAP block, if performed properly, should provide relief from this incisional pain. Since this patient had underlying chronic inflammatory bowel disease, it is difficult to know whether the persistent pain was due to an ineffective TAP block or due to her underlying, preexisting disease process. Since the patient's pain continued, the surgeon requested a CTE. Providers often feel "handcuffed" or that a "bridge is burned" once liposome bupivacaine is used because of the FDA warning and are not comfortable utilizing local anesthetics in a nerve block to alleviate pain, as there is little to no data regarding this practice. With full understanding of the FDA package insert for liposome bupivacaine specifically warning against the use of additional bupivacaine and other amide-type local anesthetics for 96 hours after the use of liposome bupivacaine, we cautiously decided to proceed with a CTE. We reexamined the research presented earlier and reviewed newer studies. We discussed the plan with the patient, the surgeon, and the pharmacy; and with the experience of last year's case report in our institution, we felt confident we could safely administer a CTE to assist with the significant pain the patient was experiencing.

In addition to pain control, the CTE would also assist in reducing the risk of venous thromboembolism by preventing prolonged immobilization. Inflammatory bowel disease patients have a two- to threefold increased risk of developing deep vein thrombosis and pulmonary embolism compared with the general population, which represents a significant cause of morbidity and mortality. ${ }^{18}$ She was already receiving 
a daily prophylactic dose of low-molecular-weight heparin and in accordance with the American Society of Regional Anesthesia guidelines, we ensured that 12 hours had passed since her last dose of low-molecular-weight heparin before finally placing the CTE. ${ }^{19}$

In this specific case report, postoperatively, our patient received a total of $128 \mathrm{mg}$ of bupivacaine through her CTE over a course of 20.5 hours between the start of the infusion and the blood draw for the second plasma level. The plasma concentrations were not available at the time of CTE administration because the labs were outsourced for processing, and results were unavailable for approximately 3 days. Plasma concentrations were acquired to verify the safety profile with escalating doses of bupivacaine following liposome bupivacaine administration. The FDA package insert allows for use of liposome bupivacaine following bupivacaine $\mathrm{HCl}$ if the dose in milligram of the bupivacaine $\mathrm{HCl}$ remains $<50 \%$ of the dose of liposome bupivacaine given. However, the use of other bupivacaine formulations following liposome bupivacaine administration has not been studied. ${ }^{5}$ As shown in Figure 4, neurotoxic and cardiotoxic plasma levels of bupivacaine begin at $2.0 \mu \mathrm{g} / \mathrm{mL}$ and $4.0 \mu \mathrm{g} / \mathrm{mL}$, respectively. ${ }^{7,11,20}$ The plasma levels of bupivacaine in this patient remained well below the threshold for potential toxicity, both pre- and post-epidural administration as hypothesized, $0.2 \mu \mathrm{g} / \mathrm{mL}$ and $0.7 \mu \mathrm{g} / \mathrm{mL}$, respectively. Additionally, no toxic effects were observed during the postoperative period.

Our measurements may even be an overestimation of the patient's actual bupivacaine concentrations. The majority (ie, $>90 \%$ ) of bupivacaine in the plasma is bound to AGP, an acute phase reactant, which is upregulated postoperatively. ${ }^{20}$ Presuming that the concentration of unbound (pharmacologically

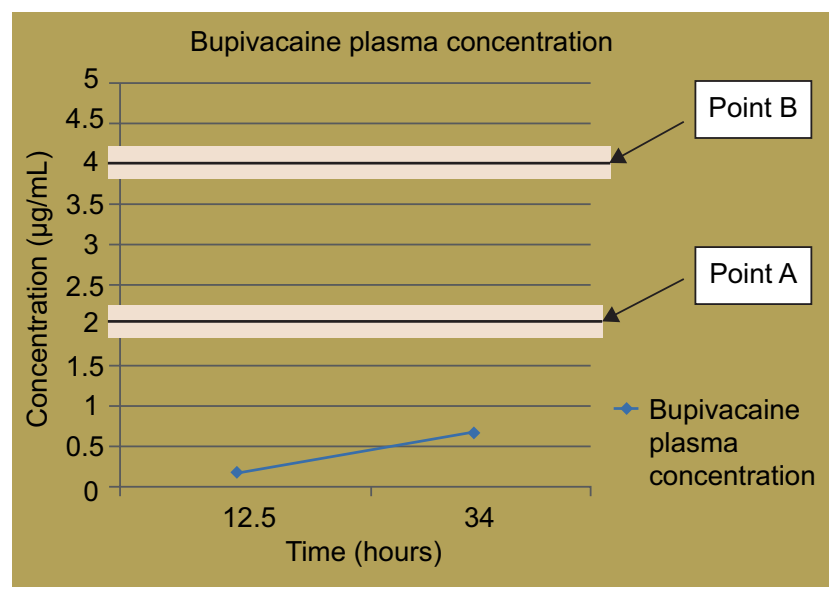

Figure 4 Graph illustrates two points of toxicity.

Notes: Point $A$ is the concentration of bupivacaine in which neurotoxic effects are normally seen. Point $B$ is the concentration of bupivacaine in which cardiotoxic effects are normally seen. The blue data points represent the patient's measured bupivacaine plasma concentration levels and corresponding postoperative times. active) bupivacaine is responsible for systemic toxic effects, the influence of protein binding should be taken into account. With more AGP, there should be more protein binding of bupivacaine, leading to a reduction in pharmacologically active bupivacaine, and we should therefore be further away from toxic bupivacaine levels. In this case report, total bupivacaine concentrations were collected (ie, protein-bound and free pharmacologically active bupivacaine).

\section{Conclusion}

The combined use of liposome bupivacaine and a bupivacaine CTE infusion in the postoperative management of this patient demonstrated no safety concerns, provided excellent analgesia and plasma concentrations of bupivacaine remained far below toxic levels. Further studies need to be conducted in order to determine the margin of safety when using liposome bupivacaine with other bupivacaine formulations and delivery methods.

\section{Disclosure}

The authors report no conflicts of interest in this work.

\section{References}

1. Hall MJ, DeFrances CJ, Williams SN, Golosinskiy A. National hospital discharge survey: 2007 summary. Natl Health State Report. 2010;24:1-20.

2. Apfelbaum JL, Chen C, Mehta SS, Gan TJ. Postoperative pain experience: results from a national surgery suggest postoperative pain continues to be undermanaged. Anesth Analg. 2003;97:534-540.

3. Wells N, Pasero C, Margo M. Improving the quality of care through pain assessment and management. In: Hughes RB, editor. Patient Safety and Quality: An Evidence-Based Handbook for Nurses, vol 1. Rockville, MD: Agency for Healthcare Research and Quality; 2008: 469-497.

4. American Society of Anesthesiologists Task Force on Acute Pain Management. Practice guidelines for acute pain management in the perioperative setting: an updated report by the American Society of Anesthesiologists Task Force on Acute Pain Management. Anesthesiology. 2004;100(6):1573-1581.

5. Exparel [package insert]. San Diego, CA: Pacira Pharmaceuticals Inc; 2011.

6. Pacira Pharmaceuticals, Inc. Available from: www.pacira.com. Accessed November 4, 2011.

7. Portillo J, Kamar N, Melibary S, Quevedo E, Bergese S. Safety of liposome extended-release bupivacaine for postoperative pain control. Front Pharmacol. 2014:5:90.

8. Dasta J, Ramamoorthy S, Patou G, Sinatra R. Bupivacaine liposome injectable suspension compared with bupivacaine $\mathrm{HCl}$ for the reduction of opioid burden in the postsurgical setting. Curr Med Res Opin. 2012:28(10):1609-1615.

9. Gorfine S, Onel SE, Patou G, Krivokapic Z. Bupivacaine extendedrelease liposome injection for prolonged postsurgical analgesia in patients undergoing hemorrhoidectomy: a multicenter, randomized, double-blind, placebo-controlled trial. Dis Colon Rectum. 2011:54(12):1552-1559.

10. Morales R Jr, Mentz 3rd H, Newall G, Patronella C, Masters O 3rd. Use of abdominal field block injections with liposomal bupivacaine to control postoperative pain after abdominoplasty. Aesthet Surg J. 2013:33(8):1148-1153. 
11. Singla N, Somasundaram L, Gadsden J, Kramer W, Hadzic A. Exparel ${ }^{\mathrm{TM}}$, A bupivacaine extended- release multivesicular liposomal formulation, exhibits pharmacokinetic properties consistent with sustained release characteristics. ASA poster presentation; 2009; New Orleans, LA.

12. Barash PG, Cullen BF, Stoelting RK, Cahalan M. Clinical Anesthesia. 7th ed. Philadelphia, PA: Wolters Kluwer Health/Lippincott Williams \& Wilkins; 2013: 572-575.

13. Bramlett K, Onel E, Viscusi ER, Jones K. A randomized, doubleblind, dose-ranging study comparing wound infiltration of DepoFoam bupivacaine, an extended-release liposomal bupivacaine, to bupivicane $\mathrm{HCl}$ for postsurgical analgesia in total knee arthroplasty. Knee. 2012;19(5):530-536.

14. Jorfeldt L, Lofstrom B, Pernow B, Persson B, Wahren J, Widman B. The effect of local anesthetics on the central circulation and respiration in man and dog. Acta Anaesthesiol Scand. 1968;12(4):153-169.

15. Munson ES, Tucker WK, Ausinsch B, Malagodi MH. Etidocaine, bupivacaine, and lidocaine seizure thresholds in monkeys. Anesthesiology. 1973;42(4):471-478.
16. Emanuelsson BM, Zaric D, Nydahl PA, Axelsson KH. Pharmacokinetics of ropivicaine and bupivacaine during 21 hours of continuous epidural infusion in healthy male volunteers. Anesth Analg. 1995;81(6):1163-1168.

17. Terrien BD, Slotto J, Connolly N, DaValle B. Safe, Simultaneous use of liposomal bupivacaine in the surgical wound and a bupivacaine epidural for postoperative analgesia. ASA poster presentation; 2014; San Diego, CA.

18. Yuhara H, Steinmaus C, Corley D, Koike J, Igarashi M, Suzuki T, Mine T. Meta-analysis: the risk of venous thromboembolism in patients with inflammatory bowel disease. Aliment Pharmacol Ther. 2013;37(10): 953-962.

19. Horlocher TT, Wedel DJ, Rowlingson JC. Regional anesthesia in the patient receiving antithrombotic or thrombolytic therapy: American Society of Regional Anesthesia and Pain Medicine Evidence Based Guidelines (Third Edition). Reg Anesth Pain Med. 2010;35(1):64-101.

20. Wulf H, Winckler K, Maier C, Heinzow B. Pharmacokinetics and protein binding of bupivacaine in postoperative epidural analgesia. Acta Anaesthesiol Scand. 1988;32(7):530-534.
Journal of Pain Research

\section{Publish your work in this journal}

The Journal of Pain Research is an international, peer reviewed, open access, online journal that welcomes laboratory and clinical findings in the fields of pain research and the prevention and management of pain. Original research, reviews, symposium reports, hypothesis formation and commentaries are all considered for publication.

\section{Dovepress}

The manuscript management system is completely online and includes a very quick and fair peer-review system, which is all easy to use. Visit http://www.dovepress.com/testimonials.php to read real quotes from published authors. 